\title{
Specific mass increment and nonequilibrium crystal growth
}

\author{
Leonid M. Martyushev $^{\mathrm{a}, \mathrm{b}, *}$, Pavel S. Terentiev ${ }^{\mathrm{a}}$ \\ a Ural Federal University, 19 Mira St., Ekaterinburg, 620002, Russia \\ ${ }^{\mathrm{b}}$ Institute of Industrial Ecology, Russian Academy of Sciences, 20 S. Kovalevskaya St., 620219 Ekaterinburg, Russia
}

\section{H I G H L I G H T S}

- Specific mass increment of seaweeds is described well with the power law.

- This power law is similar to the dependence for the dendrites.

- The total growth time can be used for writing the universal curve of unsteady growth.

\section{A R T I C L E I N F O}

\section{Article history:}

Received 26 January 2013

Received in revised form 1 April 2013

Available online 23 April 2013

\section{Keywords:}

Nonequilibrium crystallization

Specific mass increment

Growth curves

Dendrite

Seaweed

\begin{abstract}
A B S T R A C T
Unsteady nonequilibrium crystallization of ammonium chloride from an aqueous solution resulting in the formation of irregular, so-called seaweed, structures is experimentally investigated. It is shown that specific increment of mass for the coexisting structures (or parts thereof) is the same and changes with time $(t)$ according to the power law $a / t-b$, where the factor $a=1.87 \pm 0.09$ and the factor $b$ is determined by the system relaxation time. The normalization of the power law to the total time of structure growth allows obtaining a universal law that describes the specific mass increment with time for both seaweed and dendrite structures (including the non-coexisting ones).
\end{abstract}

(c) 2013 Elsevier B.V. All rights reserved.

\section{Introduction}

If nuclei of a new crystalline phase originate in a sufficiently supersaturated solution/vapor (or a supercooled melt), then eventually, during the relaxation of the system to the equilibrium, they will grow into different nonequilibrium structures: dendrites, nonsymmetrical diffusion-limited aggregates (DLA), seaweed structures, etc. [1-6]. This phenomenon is very common both in nature (for example, during the formation of snow crystals in clouds) and in industry (specifically in metallurgy, in the case of steel production). As a consequence, this phenomenon has been comprehensively studied in the scientific literature for at least the last fifty years [7-12]. It is well known that the nonequilibrium growing structures are extremely inhomogeneous, specifically they consist of crystal tree-like branches which have various sizes and growth rates. Additionally, in the case of such growth, the coexistence of principally different structures (for example, regular and irregular dendrites) is also possible [5,6]. The above phenomena are observed even if the degree of nonequilibrium during crystallization is maintained artificially. If crystallization is considered subject to the reduction of the degree of nonequilibrium in the medium caused by the free crystal growth (the most natural and common case in nature), then the morphology and kinetics of the developed structures will become even more complex. One of the most intriguing questions that arise here is as follows: is there any quantity that is the same (invariant) for different parts of nonequilibrium crystallizing structures? Obviously, if any common features between so complex and diverse (in terms of kinetics

\footnotetext{
* Corresponding author at: Ural Federal University, 19 Mira St., Ekaterinburg, 620002, Russia.

E-mail addresses: mlm@ecko.uran.ru, leonidmartyushev@gmail.com (L.M. Martyushev).
} 


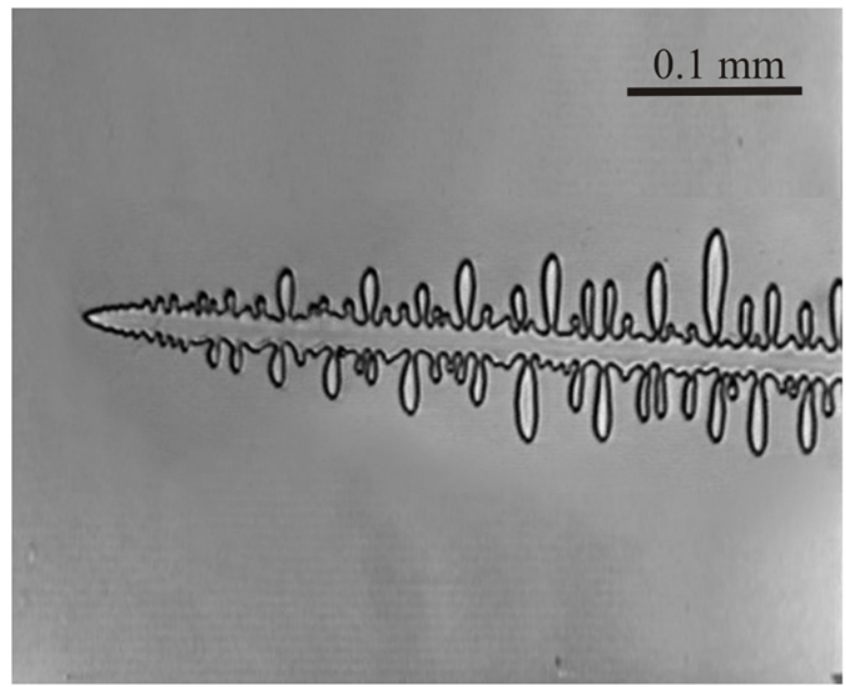

Fig. 1. Example of a dendrite in the case of ammonium chloride crystallization.

and morphology) nonequilibrium growing crystals and/or parts thereof are found, this would be of high practical and fundamental importance. Until recently, in the case of the steady dendrite growth, the quantity equal to the product of the dendrite tip growth rate and the squared radius of its curvature was considered as such a universal parameter [8-12]. This quantity did not depend on the degree of nonequilibrium in the medium and was determined by the physical and chemical properties of the crystallized matter only. However, lately, after a number of rigorous experiments, doubts have been cast on the universality of this quantity (see e.g. Ref. [13]).

Not so long ago, the paper [14] advanced a hypothesis that the specific mass increment (normalized increment of crystal mass is the synonym) in the case of the steady and unsteady dendrite growth (Fig. 1) can represent some universal quantity. This hypothesis was experimentally confirmed during the study of ammonium chloride crystallization from a supersaturated aqueous solution in a thin flat capillary. Let us briefly present the main conclusions of that study: (1) The specific increment of mass (area in the considered quasi-two-dimensional system) is the same for different coexisting dendrite branches (primary and side) and changes with time ( $t$ ) under the power law $a / t-b$ (the time is counted from the start of structure observation). (2) The factor $a$ depends on the physical and chemical properties of the crystallized matter and the geometry of the area where the mass increment of the branch is measured (for dendrites of ammonium chloride crystallized from an aqueous solution [14], this factor is equal to $1.7 \pm 0.2$ ). The factor $b$, being inversely proportional to the dendrite growth time (system relaxation time), depends on the degree of unsteadiness of the considered growth (for the steady process $b=0$ ). As a consequence, even for non-coexisting ${ }^{1}$ growing primary/side branches of the dendrites with the same chemical composition, the dependency of the specific mass (area) increment of branches on time has a form similar (accurate within an additive constant) to this: $a / t$.

It is known $[6,14]$ that the so-called irregular (seaweed) structures ${ }^{2}$ (Fig. 2) representing a less common form of growth are observed, besides the dendrites (Fig. 1), during the crystallization of ammonium chloride. As can well be seen, these structures essentially differ from the dendrite ones both in terms of the ratio of the central branch area to the sidebranch area and in terms of their symmetry. Will the specific area increment of individual parts (sidebranches) of seaweed structures evolve in accordance with the law $a / t$ ? What will the factor $a$ be equal to? It is extremely interesting and important to know the answers to these questions in order to understand the fundamental laws of relaxation of nonequilibrium crystal systems. The search for these answers forms the subject of the present paper.

\section{Experimental procedure}

The experimental procedure, the specifics of video processing of the obtained structures, and the analysis of errors are set forth in detail in the recently published paper [14]. Let us repeat only some important points.

1. $\mathrm{The}_{\mathrm{NH}} \mathrm{Cl}$ crystallization from the aqueous solution took place in the thin flat capillary, which thickness did not exceed $0.05 \mathrm{~mm}$. For this purpose, the solution pre-saturated at the temperature of $35^{\circ} \mathrm{C}$ was rapidly cooled to the temperature of

\footnotetext{
1 This implies branches either growing at substantially different times or branches growing simultaneously but having essentially different stages of growth due to a lot of reasons (times and conditions of origin, mutual influence ("struggle") of branches, etc.).

2 Such structures are referred to as "fractal seaweed" in Ref. [3] and "seaweed" in Ref. [4]. Let us note that these structures are called "irregular patterns" in the paper [6].
} 


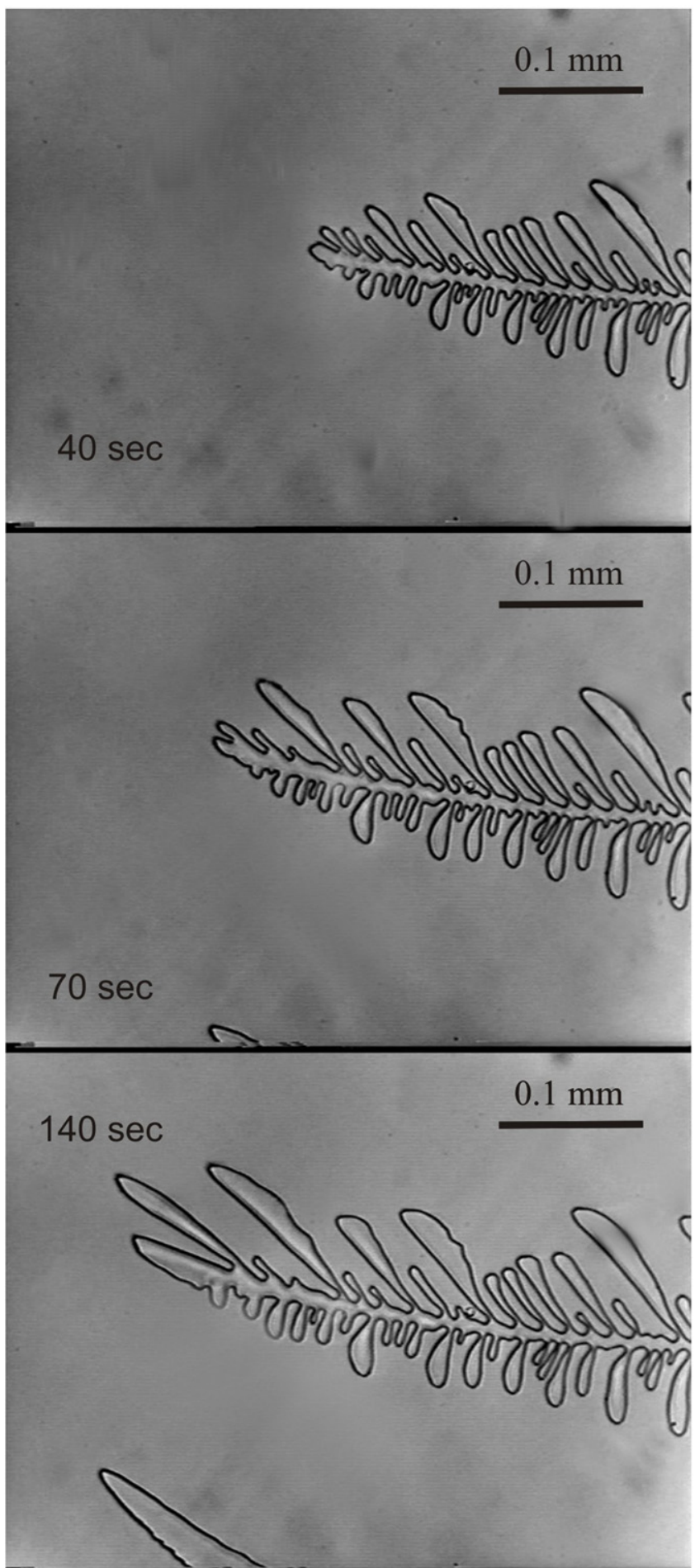

Fig. 2. Frames of a seaweed of ammonium chloride growing from the aqueous solution. Starting approximately from the 70th second after the start of observation, the visual field captures a growing dendrite that coexists with the observed seaweed structure.

$20^{\circ} \mathrm{C}$ within 2-3 s. This caused the appearance and growth of multiple crystals. As a result, the supersaturation occurred due to the rapid cooling was removed. We studied only this active nonequilibrium stage of the crystal growth (the subsequent slow relaxation of dendrites to the quasi-equilibrium crystal shapes was not considered). The main body of experiments was conducted in the similar manner. The increase or decrease of the upper or lower temperature by at most five degrees 


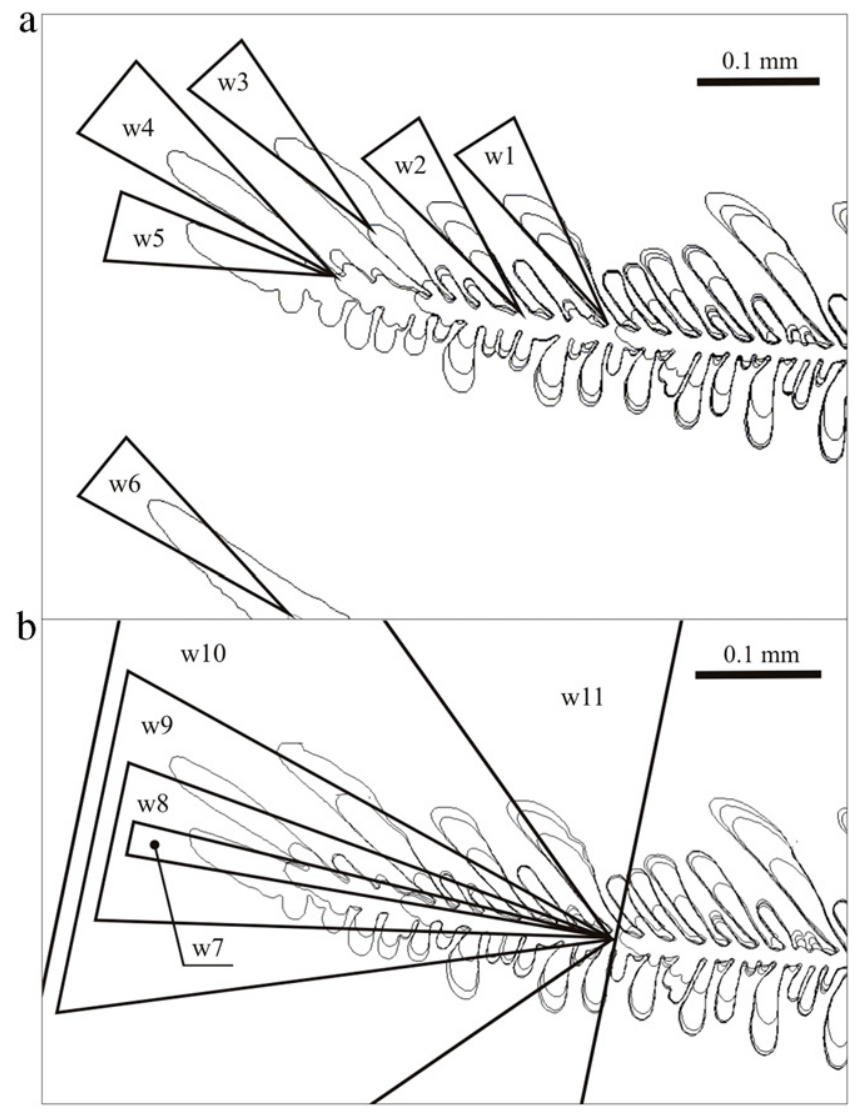

Fig. 3. Sectors used for area measurement. (a) Sizes of all sectors $w 1-w 6$ are $0.1 \pi$ rad. The sectors $w 1, w 2$ as well as the sectors w3-w6 allow measurement of the simultaneous growth of the branches contained therein; the sectors w1, w2 and the sectors w3-w6 are separated by the time interval of 70 s. (b) Sizes of sectors (in radians): $\mathrm{w} 7=0.02 \pi, \mathrm{w} 8=0.1 \pi, \mathrm{w} 9=0.2 \pi, \mathrm{w} 10=0.5 \pi, \mathrm{w} 11=\pi$. These sectors of different sizes are used to measure the simultaneous growth of the branches contained therein.

failed to produce any notable changes in the results. The observations and measurements were not performed outside this range of temperatures.

2. Only single freely growing crystals, which were separated from the neighboring crystals and from the borders of the crystallization cell by at least the diffusion length, were targeted for observation and recording (usually at 110-fold magnification). We studied at about 100 growing crystals. In the vast majority of cases, the dendrite growth was observed (Fig. 1). The so-called irregular patterns (seaweeds) were observed in approximately $5 \%$ of the cases (Fig. 2).

3. The area $(S)$ of the seaweed projection onto the cell surface was measured. This value is directly proportional to the crystal mass. Indeed, the observed seaweeds are three-dimensional; however, their branches are almost parallel to the observation plane of the thin flat capillary. As a conclusion, the area of the seaweed projection onto the cell surface is proportional to the volume (if the crystal branches have approximately identical thickness) or the mass (if the crystal density is constant) of the observed crystal.

4. The area $S$ was measured relative to the time $t$ at least once per second in triangular sectors located along the direction of growth of the whole crystal and its sidebranches (Fig. 3); the errors of determination of $S(t)$ and the specific increment $S^{\prime}(t) / S(t)$ (where $S^{\prime}$ is the change of the crystal area with time) did not exceed $20 \%$.

5. The results obtained during the analysis of one of the seaweeds (Fig. 2) are given below. The results for other seaweeds are similar within the error limits.

\section{Results and discussion}

The main results of the measurement are given in Figs. 4-6. Based on their analysis, it is possible to draw the following conclusions:

1. For the simultaneously growing different parts of the crystal (branches), the dependencies of area on time (Fig. 4(a), Fig. 5(a), and Fig. 6(a)) can differ both in terms of quantity (see the sectors w3 and w5, or w7 and w8) and in terms of quality (for the sector w5, the dependency reaches the saturation stage, which is not the case for w4). In the case of the simultaneously growing branches of the crystal, the qualitative difference of $S(t)$ indicates that these branches are at 

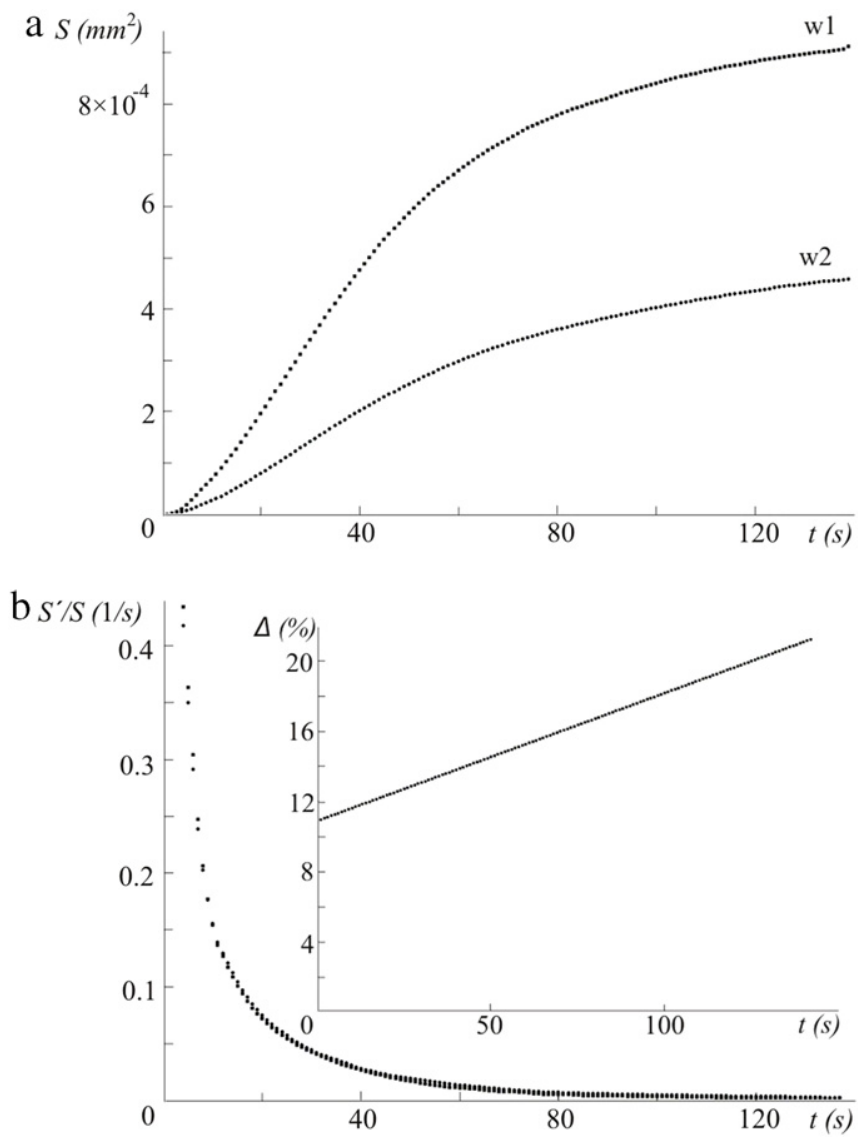

Fig. 4. Measurement results for the simultaneously growing seaweed sidebranches. (a) Values of the area $S$ relative to the growth time $t$ for the sectors $w 1$ and w2 (Fig. 3a). (b) Values of the specific area increment $S^{\prime}(t) / S(t)$ relative to the growth time $t$ for w1 and w2. The inset shows the relative difference of $S^{\prime}(t) / S(t)$ for $w 1$ and $w 2$.

Table 1

Values of the two-parameter approximation $a / t-b$ of $S^{\prime}(t) / S(t)$ for the Fig. 3(a) sectors.

\begin{tabular}{lllllll}
\hline Factors & Sectors & & & \\
\cline { 2 - 6 } & w1 & w2 & w3 & w4 & w5 & w6 \\
\hline$a$ & $1.87 \pm 0.10$ & $1.92 \pm 0.10$ & $1.91 \pm 0.08$ & $1.89 \pm 0.08$ & $1.85 \pm 0.09$ & $1.80 \pm 0.09$ \\
$b, \times 10^{-2}(1 / \mathrm{s})$ & $1.5 \pm 0.1$ & $1.6 \pm 0.4$ & $1.4 \pm 0.2$ & $0.8 \pm 0.2$ & $1.3 \pm 0.2$ & $0.7 \pm 0.2$ \\
\hline
\end{tabular}

different growth stages (e.g., the branch in the sector w5 starts decelerating in $30 \mathrm{~s}$ after the start of observation, whereas such deceleration in the sector w4 takes place later, outside the observation time interval). Such a difference in the behavior of the branches is connected with a lot of factors such as: time of origin, difference in growth conditions (due to a difference in supersaturation, impurities, surrounding branches, etc.). The branches growing at the same time but being at different development stages are not be considered as the coexisting ones. The "struggle" for existence between such branches is not a rare case; as a result, one of the branches may suppress the growth of the other. Such behavior of branches was also observed in the case of the dendrite growth [14], but it is more common for the irregular structures under consideration.

2. Despite a considerable difference in $S(t)$ for coexisting and non-coexisting (including non-simultaneously growing) branches of the structure under study, the specific area increment with time $S^{\prime}(t) / S(t)$ has a form similar ${ }^{3}$ (accurate within an additive constant) to this: $a / t$. Additionally, for all the studied sectors (w1-w11), the factor $a$ agreed within the confidence interval (see Tables 1 and 2). If the factor $a$ is averaged in the context of the hypothesis of its normal distribution, its value will be $1.87 \pm 0.09$, which agrees, within the confidence interval, with the result $a=1.7 \pm 0.2$ obtained for a similar crystallization system in the case of the dendrite growth [14].

3. The specific mass increment $S^{\prime}(t) / S(t)$ is described with the dependency $a / t-b$ (with the correlation factor above 0.99). For the coexisting branches, the increments $S^{\prime}(t) / S(t)$ agree (within the experiment error) at each moment of time,

\footnotetext{
3 It should be noted that the time counted from the start of structure observation is assumed to be $t$. In the case of the simultaneous measurement of the specific increment for different branches, the count reference time is the same; in the case of the non-simultaneous measurement, it is different.
} 

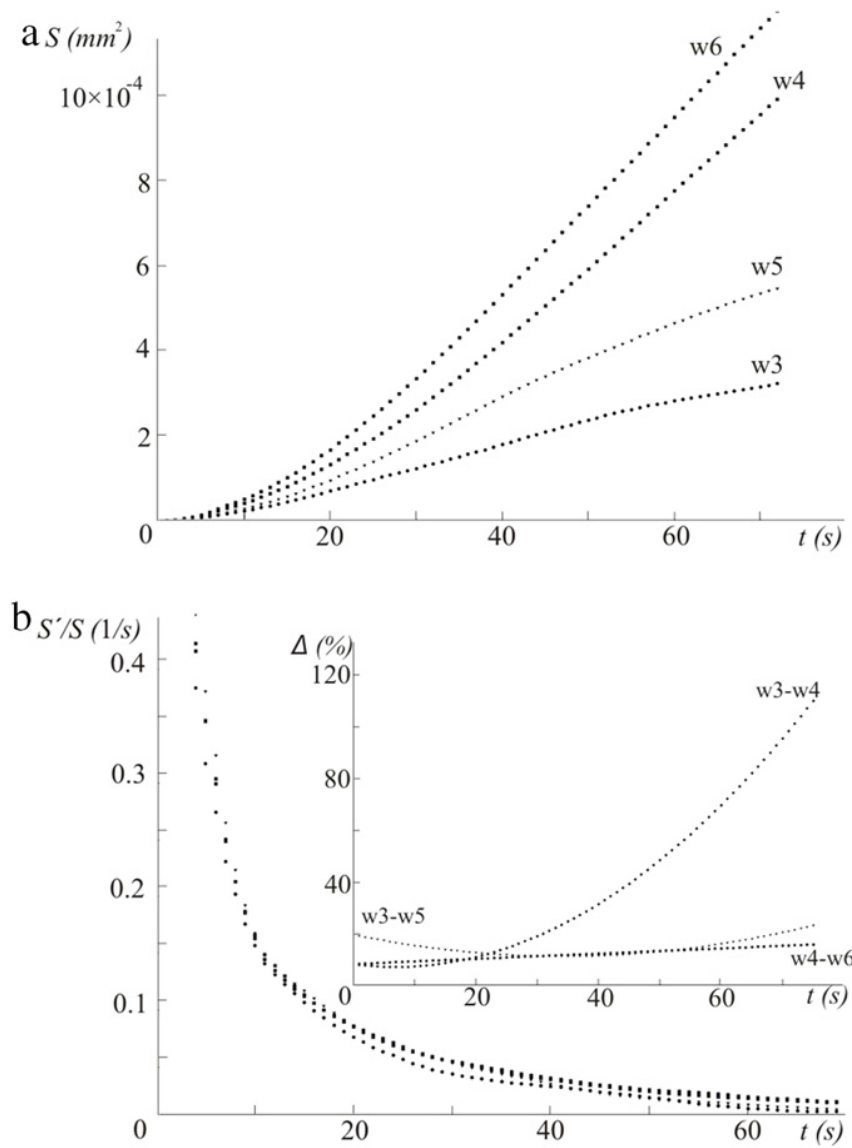

Fig. 5. Measurement results for the simultaneously growing seaweed sidebranches. (a) Values of the area $S$ relative to the growth time $t$ for the sectors w3-w6 (Fig. 3a). (b) Values of the specific area increment $S^{\prime}(t) / S(t)$ relative to the growth time $t$ for w3-w6. The inset shows the relative difference of $S^{\prime}(t) / S(t)$ for three pairs: w3 and w5, w3 and w4, and w4 and w6.

Table 2

Values of the two-parameter approximation $a / t-b$ of $S^{\prime}(t) / S(t)$ for the Fig. 3(b) sectors.

\begin{tabular}{llllll}
\hline Factors & \multicolumn{3}{l}{ Sectors } & & \\
\cline { 2 - 5 } & w7 & w8 & w9 & w10 & w11 \\
\hline$a$ & $1.87 \pm 0.08$ & $1.93 \pm 0.12$ & $1.94 \pm 0.06$ & $1.85 \pm 0.05$ & $1.74 \pm 0.14$ \\
$b, \times 10^{-2}(1 / \mathrm{s})$ & $1.0 \pm 0.1$ & $1.1 \pm 0.2$ & $1.2 \pm 0.1$ & $1.0 \pm 0.1$ & $1.1 \pm 0.3$ \\
\hline
\end{tabular}

despite a considerable quantitative difference between the values of $S(t)$ (see Fig. 4 (a) and Fig. 5(a)). Thus, the agreement is observed for the following pairs of coexisting branches: w1 and w2; w3 and w5; w4 and w6 (see Fig. 4(b), Fig. 5(b)). As a consequence, the values of the factors $b$ for these pairs agree within the error limits (see Table 1). For the non-coexisting structures, there is a considerable difference between the values of $S^{\prime}(t) / S(t)$; thus, for the branches in the sectors w3 and $\mathrm{w} 4$, the relative difference between these values can reach more than $100 \%$ (see the inset in Fig. 5(b)). Correspondingly, the values of the factor $b$ differ for the non-coexisting branches. According to the results [14], the value of this factor is inversely proportional to the active growth time of the branch. The example under consideration confirms this regularity. So, for the branch w3, growth time is about $40 \mathrm{~s}$ (for definiteness, the inflection point of the curve $S(t)$ is used) and $b=0.014 \pm 0.002$; whereas for the branch $\mathrm{w} 4$, the growth time is more than $70 \mathrm{~s}$ and $b=0.008 \pm 0.002$.

4. The above regularities do not depend on the size of the sector where the observation is performed. This is illustrated in Fig. 6(b). For different sizes of the sectors located along the direction of structure growth, the specific mass increments agree within the experiment error (20\%). Correspondingly, the values of the factors $a$ and $b$ (Table 2) agree for these sectors.

Thus, for both regular (dendrite) and irregular (seaweed) structures in the case of the nonequilibrium crystallization in the $\mathrm{NH}_{4} \mathrm{Cl}-\mathrm{H}_{2} \mathrm{O}$ system at hand, the specific mass increment is described with the simple dependency $a / t-b$. Accordingly, the behavior of mass (area in the quasi-two-dimensional case at hand) relative to time, accurate within a constant (normalization) factor, has the form $t^{a} \mathrm{e}^{-b t}$. This function describes the growth curves observed during the experiment (Fig. 4(a), Fig. 5(a), and Fig. 6(a)) rather well. Let us further refer to it as the DS function/ model (the initial letters of 

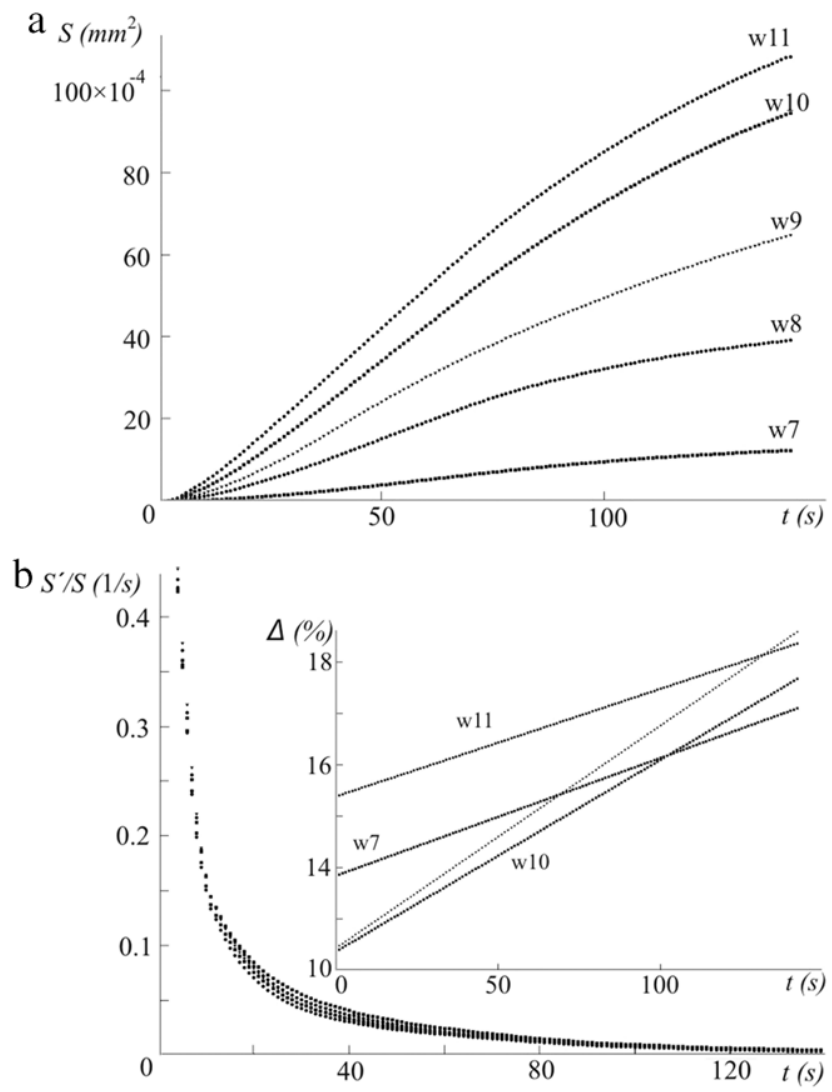

Fig. 6. Measurement results in the sectors of different sizes. (a) Values of the area $S$ relative to the growth time $t$ for the sectors w7-w 11 (Fig. $3 b$ ). (b) Values of the specific area increment $S^{\prime}(t) / S(t)$ relative to the growth time $t$. The inset shows the relative difference between $S^{\prime}(t) / S(t)$ for the sectors w7, w9-w11, and $S^{\prime}(t) / S(t)$ for w8.

"dendrite" and "seaweed" were taken for naming). However, other models are often used in the literature to describe the dependency of mass on time for nonequilibrium growing structures ${ }^{4}$ : Logistic : $1 /\left(1+\exp \left(-k\left(t-t_{m}\right)\right)\right)$, Richards : $1 /\left(1+n \exp \left(-k\left(t-t_{m}\right)\right)\right)^{1 / n}$, Gompertz : $\exp \left(-\exp \left(-k\left(t-t_{m}\right)\right)\right)$, and Weibull : $1-\exp \left(-k t^{n}\right)(\operatorname{see}$ e.g. Refs. [15, 16]). The obtained experimental values of $S(t), S^{\prime}(t) / S(t)$ were approximated in the MATLAB (curve fitting toolbox) with these functions, where the factors $k, n$ and $t_{m}$ were used as free (adjustable) parameters. It is revealed that the Gompertz and Logistic models are not suitable for description of the experiment, and the Weibull and Richards models describe the experiment slightly worse than the DS model at the end of the growth time. Furthermore, these alternative models are more mathematically complex compared to the DS model. Apart from the mentioned disadvantage, the alternative models under study have one key peculiarity. So, according to these models, the time at which the structure growth stops proves to be equal to infinity (the growth rates asymptotically tend to zero values). Obviously, this is not true for real growth processes: any structure exists for a finite lifetime having its definite moments of "birth" (origin) and "death" (stop). As opposed to these alternative models, the DS model $\left(t^{a} \cdot \mathrm{e}^{-b t}\right)$ reaches the zero rate at the moment of time $t^{*}=a / b\left(S^{\prime}\left(t^{*}\right)=0\right)$. After this time, the DS model demonstrates the decrease in mass of the dendrite/seaweed branches. This is observed when a supersaturation in a solution is removed. As a result, the dendrites/seaweeds transform with time into equilibrium shapes (for ammonium chloride crystals, this shape is round). The possibility of quantitative description of this second stage using the DS model should be studied further.

Thus, the lifetime is a critical parameter; therefore, we use it as a scale for the time nondimensionalization $\left(\tilde{t}=t / t^{*}\right)$. In this case, the dimensionless specific increment of crystal mass $\tilde{S^{\prime}} / \tilde{S}$ can be written in the form $a \cdot(1 / \tilde{t}-1)$. Fig. 7 gives this nondimensionalized data $a \cdot(1 / \tilde{t}-1)$ for the seaweeds and dendrites. For clarity, the graph is constructed using the logarithmic converted system of coordinates, where the nondimensional specific increment $1.8 \cdot(1 / \tilde{t}-1)$ is a linear function. It is seen (Fig. 7) that all nondimensional curves are rather close (within $20 \%$ error limits) to the universal curve: $1.8 \cdot(1 / \tilde{t}-1)$. The presented results (Fig. 7 ) indicate that the obtained experimental data regarding the time dependence of the specific area increment can be described well using the simple one-parameter dependences after the corresponding

\footnotetext{
4 The functions of the models are given up to a constant factor. The factors $k$ and $n$ determine the type of growth curvature, and $t_{m}$ is the time required for the structure to reach the maximum rate.
} 


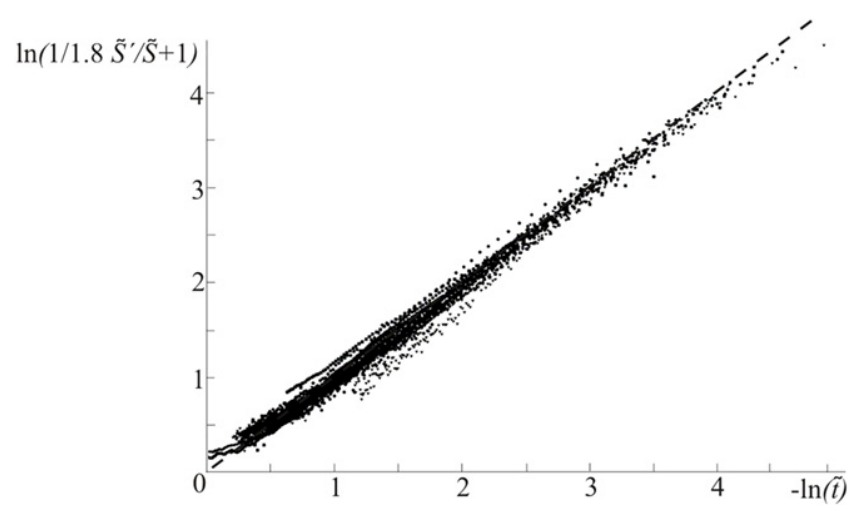

Fig. 7. Values of the nondimensional specific mass increments for seaweed in Figs. 4-6 and for dendrites in Figs. 5 and 6 [14] relative to the nondimensional time. The nondimensionalization and the conversion of coordinate axes were performed according to the rules given in the paper text. The dashed line corresponds to $1.8 \cdot(1 / \tilde{t}-1)$. The graph summarizes the results previously obtained for the dendrites [14] (their number is 17 , see Table 2 [14]) and the data obtained for the seaweeds herein (their number is 11 , see Tables 1 and 2 ).

nondimensionalization. Thus, the scaling with $t^{*}$ gives evidence of the similarity of all dependencies $\tilde{S^{\prime}} / \tilde{S}$ observed in the case of crystallization of ammonium chloride.

\section{Conclusion}

As a result of the conducted experiments on the free nonequilibrium growth of ammonium chloride crystals from the aqueous solution, it was established that the specific mass (area) increment of seaweeds and dendrites is described well with the function $a / t-b$. For all considered seaweed crystals, the factor $a$ assumes the values of $1.87 \pm 0.09$, which agrees with the value previously determined for the dendrite growth $(1.7 \pm 0.2)$ [14] within the confidence interval. The factor $b$ depends on the relaxation time of the unsteady system at hand and shows the same value for the coexisting structures. The finite crystallization time is an important characteristic of the used DS model $(a / t-b)$. This time can be used for writing the universal curve (in dimensionless variables) for the specific mass increment of the structure (or a part thereof) in the case of the unsteady crystallization in the $\mathrm{NH}_{4} \mathrm{Cl}-\mathrm{H}_{2} \mathrm{O}$ system. The obtained results give rise to new questions. Specifically, will the determined universal dependency describe specific mass increment in the case of other crystallization (growth) systems? Another question interesting and important from the fundamental and applied perspective: how well does the found DS model allow predicting the whole life cycle of the crystal based on some small number of known (measured) values of the crystal mass at some moments of time? The given points provide wide space for further study of free nonequilibrium growth of various structures.

\section{References}

[1] J. Feder, Fractals, Plenum Press, NY, London, 1988.

[2] E. Ben-Jacob, From snowflake formation to the growth of bacterial colonies, part I: diffusive patterning in non-living systems, Contemp. Phys. 34 (1993) 247.

[3] T. Ihle, H. Müller-Krumbhaar, Fractal and compact growth morphologies in phase transitions with diffusive transport, Phys. Rev. E 49 (1994) 2972.

[4] H.M. Singer, J.H. Bilgram, Quantitative description of morphological transitions in diffusion-limited growth of xenon crystals, Phys. Rev. E 70 (2004) 031601.

[5] H. Honjo, S. Ohta, M. Matsushita, Phase diagram of a growing succinonitrile crystal in supercooling-anisotropy phase space, Phys. Rev. A 36 (1987) 4555 ;

Y. Sawada, B. Perrin, P. Tabeling, et al., Oscillatory growth of dendritic tips in a three-dimensional system, Phys. Rev. A 43 (1991) 5537;

H. Honjo, S. Ohta, Dense-branching morphology of an $\mathrm{NH}_{4} \mathrm{Cl}$ crystal, Phys. Rev. A. 45 (1992) R8332.

[6] Y. Sawada, Transition of growth form from dendrite to aggregate, Phys. A 140 (1986) 134.

[7] B. Chalmers, Principles of Solidification, Wiley, NY, 1964.

[8] J.S. Langer, Instabilities and pattern formation in crystal growth, Rev. Modern Phys. 52 (1980) 1.

[9] D. Kessler, J. Koplik, H. Levine, Pattern selection in fingered growth phenomena, Adv. Phys. 37 (1988) 255.

[10] W. Kurz, D.J. Fisher, Fundamentals of Solidification, Trans. Tech. Pub., 1992.

[11] M.E. Glicksman, S.P. Marsh, in: D.T.J. Hurle (Ed.), Handbook of Crystal Growth, Vol. 1, Elsevier, 1993.

[12] M.E. Glicksman, Principles of Solidification, An Introduction to Modern Casting and Crystal Growth Concepts, Springer, 2011.

[13] M.E. Glicksman, Mechanism of dendritic branching, Metall. Mater. Trans. A 43A (2012) 391.

[14] L.M. Martyushev, P.S. Terentiev, Normalized increment of crystal mass as a possible universal parameter for dendritic growth, Phys. Rev. E 85 (2012) 041604.

[15] A. Tsoularis, Analysis of logistic growth models, Res. Lett. Inf. Math. Sci. 2 (2001) 23.

[16] R.B. Banks, Growth and Diffusion Phenomena, Mathematical Frameworks and Applications, Springer-Verlag, Berlin, 1994. 